\title{
A Proposal for Petri Net Based Web Service Application Modeling
}

\author{
Daniel Moldt, Sven Offermann, and Jan Ortmann \\ University of Hamburg, Computer Science Department \\ Vogt-Kölln-Str. 30, D-22527 Hamburg \\ \{moldt, 4offerma, ortmann\}@informatik . uni-hamburg.de
}

\begin{abstract}
Web engineering can be seen as the application of software engineering in a particular environment - the Web. Its most important aspect is the orientation towards distributed systems and their current technology. Current specification techniques like UML do not cover concurrency, distribution and deployment in a sufficient way.

We propose a modeling technique based on high-level Petri nets, called reference nets. They naturally allow for the modeling of processes. Additionally we introduce a framework that covers the other aspects of Web application modeling. Finally we introduce a tool set based on RENEW, a tool for reference nets, to support these modeling approach.
\end{abstract}

Keywords: High-level Petri nets, nets within nets, reference nets, RENEW, Web service, modeling, specification, MULAN

\section{Introduction}

Web Engineering as it is seen here is the attempt to perform high-quality software engineering in distributed systems for autonomous (often independent) participants. These systems are characterized by distribution, concurrency and often autonomy; the term Web indicates the current embedding of this process in the Internet as the underlying execution platform.

One important area in Web engineering is the area of Web services which are easier to handle than agents in general but which still contain the aspects of distribution and adaptability. Web services are self-describing, self-contained encapsulated applications. The modeling approaches currently often applied in practice are structured analysis and object-oriented analysis. The first one is generally not considered to be sufficient for future applications, while there is a large amount of research effort going into the development of the second and its main representatives the set of modeling techniques: UML [7] (Unified Modeling Language).

Especially the open problems of Web engineering, however, can not be solved by using a relatively simple concept like e.g. State charts for concurrency, autonomy and distribution. When building distributed and concurrent applications a true concurrency semantics has to be used to cover the problems which can be encountered at any time and level of the implementation. Here, an interleaving semantics is no longer sufficient. 
Distributed applications might no longer behave like an object, but might show an autonomous behavior. This leads to a modeling approach that is closely related to the agent concepts. Another aspect is the execution of distributed tasks. Here the support of workflow-like concepts is crucial. The UML does not offer a clear intuitive modeling technique for these concepts and so a lot of the specification is often implicitly done by the code.

One of the techniques that should be taken into closer consideration for modeling Web service applications are higher-level Petri nets naturally allowing to model distribution and concurrency. With MULAN [5] a proposal for the integration of agents and Petri nets has been made.

By adapting Mulan for the Web service context, we present a modeling technique that is designed to solve the central problems of Web engineering on a conceptual level. The MULAN-framework allows to build specifications that cover the above mentioned aspects of distributed applications. Our proposal extends the usual set of techniques which is available for Web engineers and provides a powerful architectural basis and structuring mechanism for web applications.

In the following section the underlying concepts and tools used in our approach are introduced. Section 3 explains the use of our approach for Web service orchestration whereas section 4 shows its application to Web service system design and adapts the existing MULAN agent framework for Web services. Section 5 summarizes the paper and gives a short outlook.

\section{Reference Nets}

The modeling approach described in the sequel is strongly supported by a tool called Renew[9]. Renew is a Petri net simulator implemented in Java which allows for the construction and the execution of reference nets.

Reference nets enrich the Coloured Petri net (CPN) formalism [4] by adding concept of nets-within-nets introduced by [10]. Nets-within-nets represent a concept of having tokens within a Petri net as net instances. This way nets can have multiple object nets within a single system net as tokens. The communication between these nets is done through synchronous channels. They allow for the exchange of parameters between the net instances and within a single net instance. This way they offer a way to communicate between net instances. Those object nets can be dynamically instantiated from a template. Multiple net instances can be generated at runtime having different parameters. By having transitions inscribed with arbitrary Java code, RENEW additionally offers an easy integration of Java and can therefore as well execute Web services.

\section{Composition of Web Services}

A common approach to build new software applications is to identify the needed functionalities that must be provided by modular units of the application and than implement and/or reuse components matching certain requirements. In the 
context of distributed systems a growing trend is to build such software components platform-independent and make them available in a distributed environment. On this basis new Web applications can be largely composed/assembled from a set of appropriate distributed components, called Web services.

A natural approach to model the order of the interactions needed to fulfill a complex business collaboration is the use of some kind of business process model. This can be described by a variety of different process markup languages. They specify the control and data flow of a business process or the interaction protocols describing the message exchange between the parties involved. A proposal for a model to specify dynamic composition of Web services based on Java, reference nets and current web languages is presented in [8], where the tasks for the realization of a new business service, like interactions or message transformations, are modeled by inscriptions of transitions in a reference net and a platform for the model based composition of Web services is implemented by the use of the Petri net simulator RENEW.

Petri nets offer a single formalism and a visual modeling technique for control flows as well as for the data flow. As a modeling technique for workflows, Petri nets have been thoroughly investigated [1]. Apart from their graphical visualization, they offer for some net variants means to verify properties of workflows like liveness or the absence of deadlocks 2].

Within the component oriented view we provide a conceptual framework and a tool set to dynamically compose Web services. Allowing for the execution of Petri nets, RENEW offers a tight integration of the specification of the control flow and its implementation. This is further supported by the formal semantics of Petri nets. As shown in [1] and demonstrated by the implementation in [3] Petri nets are powerful enough to represent all workflow concepts, which is equally true for the control flow concepts used in Web service description.

\section{Requirements for Web Service Architectures}

Besides the control flow of Web services we consider the physical or logical location of Web services to be another important area of modeling. Web services are hosted on some kind of Web server which is again located on a platform.

The access to Web services might fail or Web services might not be able to handle certain requests. In these cases the caller has to be able to dynamically switch to another service offering similar functionality. This is closely related to agents with respect to their autonomy and adaptation, what justifies a modeling approach for Web services that was adopted from the agent research area. Mulan [5] is a Petri net based modeling approach for multi-agent systems. Figure 1 depicts the basic architecture adopted for Web services. Here we see that Web services are located on some kind of physical host that they are deployed upon and that they have a logical platform (which might range over multiple hosts). The protocols specifying their behavior are again modeled as Petri nets. The ZOOM lines show a refinement from one layer to the succeeding layer. Web services might fail or might no longer be available. In these cases the caller can 


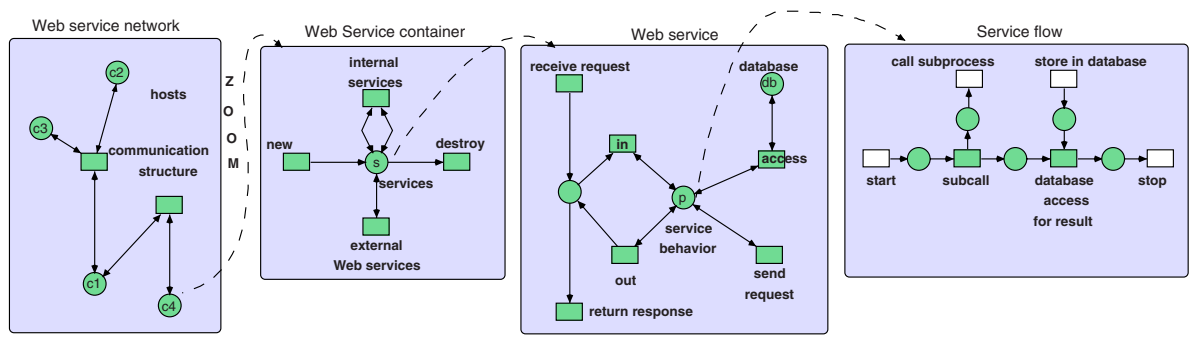

Fig. 1. Web service architecture

ask directory services on the (logical) platform for another similar service. The four layers distinguish different aspects of Web service applications.

The first layer consists of the hosts where one or more Web service containers might be deployed. These hosts offer the basic communication infrastructure.

On top of this layer we have the Web service container offering more specific communication based on SOAP and WSDL as well as access to internal services on the one hand and means to deploy and remove Web services on the other hand. A Web service implementation makes use of this layer to communicate to the outside world (e.g. to other Web services) and to internal services (e.g. databases and repositories).

The actual Web service implementation is located on top of this layer. Calls on the container are forwarded to the appropriate Web service. The Web service might then call other Web services or access internal services to handle the request.

The control flow to handle the request represents the fourth layer. An incoming request triggers a certain control flow and then passes back the results.

The architecture in figure 1 reflects the different aspects of Web services. Each of these layers can be further refined if necessary. This allows for arbitrary granularity in the modeling process. The internal and external behavior of the Web services is modeled by protocol nets. These can also be generated out of OWL-S and BPEL4WS descriptions [6].

\section{Summary and Outlook}

UML can be seen as the lingua franca. Its different modeling techniques support different views on a system. During the last years considerable effort has been put into the development of the techniques, tools and methods that are necessary for software development. A formal semantics, however, has often been neglected. and key features of current Web applications are not supported: concurrency, autonomy and adaptivity.

Even Petri nets equipped with a precise semantics do not provide sufficient means to cover these features in their models. What is missing is a software 
architecture. Here the MULAN-framework provides a conceptual and practical solution. The very high-level Petri nets are combined with agent concepts to integrate the advantages of both areas.

In this paper, a formal modeling technique has been adopted to fit the needs when modeling complex web applications. In practical work the suitability of the facets of the approach have been demonstrated. The tool set covers important aspects of web engineering. Due to the complex domain, the facets have been developed in isolation, demonstrating their respective strength. During student projects with 20 to 50 participants the approach has been applied successfully for the last three years in the area of agent-oriented software development.

We will continue to extend RENEW as the basic tool and we will focus on the Petri net aspects in combination with agents and their Web technological embedding. On the other hand we take care to use web and software engineering experience wherever it is possible. As demonstrated in [8] non-agent based structuring is investigated. In and [6] the same is shown for the Semantic web.

\section{References}

1. Wil M.P. van der Aalst and Arthur H.M. ter Hofstede. Workflow pattern homepage. Technical report, URL: http://tmitwww.tm.tue.nl/research/patterns/, 2003.

2. C. Girault and R. Valk. Petri Nets for Systems Engineering - A Guide to Modeling, Verification, and Applications. Springer-Verlag, 2003.

3. Thomas Jacob. Implementierung einer sicheren und rollenbasierten WorkflowManagement-Komponente für ein Petrinetzwerkzeug. Diplomarbeit, Universität Hamburg, Fachbereich Informatik, 2002.

4. K. Jensen. High Level Petri Nets. In A. Pagoni and G. Rozenberg, editors, Applications and Theory of Petri Nets, number 66 in Informatik Fachberichte, pages 166-180, Berlin, 1983. Springer-Verlag.

5. Michael Köhler, Daniel Moldt, and Heiko Rölke. Modelling the structure and behaviour of Petri net agents. In Proc. of 22nd International Conf. on Applications and Theory of Petri Nets 2001 (ICATPN 2001) / J.-M. Colom, M. Koutny (Eds.), Newcastle upon Tyne, UK, pages 224-242. Lecture Notes in Computer Science 2075, edited by G. Goos, J. Hartmanis and J. van Leuwen, Springer, June 2001.

6. Daniel Moldt and Jan Ortmann. DaGen: A Tool for Automatic Translation from DAML-S to High-level Petri Nets, 2004. to appear, excepted as tool paper to FASE 2004.

7. Object Management Group. UML resource page. Technical report, 2004.

8. Sven Offermann. Ein Referenz-Netz-basiertes Modell zur dynamischen Komposition von Web Services. Master's thesis, Universität Hamburg, Fachbereich Informatik, 2003.

9. Renew - the reference net workshop. URL http://www.renew.de/. Reference to the program, the source code and the documentation of the Renew simulator.

10. R. Valk. Petri nets as token objects - An introduction to elementary object nets. In J. Desel and M. Silva, editors, Proc. Application and Theory of Petri Nets, Lisbon, Portugal, number 1420 in LNCS, pages 1-25, Berlin, 1998. Springer-Verlag. 